\title{
REGIONAL DISPARITIES OF HEALTH CENTER UTILIZATION IN RURAL INDONESIA
}

\author{
*Agung Dwi Laksono ${ }^{1,2}$, Ratna Dwi Wulandari ${ }^{3}$ and Oedojo Soedirham ${ }^{4}$ \\ ${ }^{1}$ National Institute of Health Research and Development, The Ministry of Health, The Republic of Indonesia. Jl. Percetakan \\ Negara 29, Jakarta, Indonesia. \\ ${ }^{2}$ Doctoral Program, Faculty of Public Health, Universitas Airlangga, Surabaya, Indonesia. \\ ${ }^{3}$ Department of Health Policy and Administration, Faculty of Public Health, Universitas Airlangga, Surabaya, Indonesia \\ ${ }^{3}$ Department of Behavioural Science and Health Promotion, Faculty of Public Health, Universitas Airlangga, Surabaya, \\ Indonesia
}

*Corresponding Author

Agung Dwi Laksono

Email: agung.dwi.laksono-2016@fkm.unair.ac.id

\begin{abstract}
One indicator to see the quality of health system performance was to look at the disparity in the utilization of healthcare facilities. The research objective was to analyze the disparity between regions in the utilization of health centers in rural areas in Indonesia. The results of the 2013 Basic Health Survey (Riskesdas) were used as analysis material. The 2013 Riskesdas was designed a cross-sectional survey. Respondents obtained 388,598 using the multi-stage cluster random sampling method. Binary Logistic Regression Test was used to analyze data. Data is obtained through a structured questionnaire. The results showed that there were statistically significant disparities between regions. All regions showed better utilization than the Papua region as a reference. The best utilization was in the Sumatra region, which was 3.781 times more utilizing health centers than the Papua region $(O R=3.781 ; 95 \% \mathrm{Cl}=3.580-3.993)$. The utilization of health centres that approached the Papua region was the Nusa Tenggara region $(O R=1.582 ; 95 \% \mathrm{Cl}=1.490-1.679)$ and the $\mathrm{Maluku}$ region (OR $=2.175 ; 95 \%$ 1.999-2.366). All three regions are all in the Eastern part of Indonesia. The research concluded there was a disparity in health center utilization between regions in rural Indonesia. Regions in the western part of Indonesia tend to have better health center utilization in rural areas. Research results could be used as a reference for making policies that focus on equality of services to reduce existing disparities.
\end{abstract}

Keywords: the health center, utilization, region disparities, rural, Indonesia.

\section{INTRODUCTION}

Indonesia has implemented many improvements to people's access to health services. This condition has also been recognized as better than before ${ }^{1}$. Although in some cases the community still feels health services are not appropriate as expected ${ }^{2,3}$. Furthermore, the health status of the community as the outcome also varies greatly between regions ${ }^{4}$.

One indicator to see the quality of health system performance is to look at the disparity in the use of healthcare facilities. The dimension in analyzing the disparity in the use of health services that is often used is the dimensions of urban-rural, gender, socioeconomic, education, employ status, racial and ethnic, geographic, and region ${ }^{5-7}$.

Health development that has been running in Indonesia still shows disparities between urban and rural areas. Urban areas tend to have access to better health services. This condition was found because of the participation of private parties who prefer urban areas with denser population density conditions, making it more economically profitable ${ }^{8-10}$. This reason is the basis of the assumption that rural areas are more vulnerable than in urban areas.

Disparities in health services that occur between urban and rural areas contribute to the increase in the number of people suffering from chronic diseases in the countryside ${ }^{11,12}$. If allowed to continue, there will be a considerable opportunity lost that must be borne by the community and the government. In this position, the role of the Puskesmas (health center) as a gatekeeper is very important to screen patients at the basic service level ${ }^{13-15}$.

The disparity in the utilization of health care facilities is allegedly not only in the urban-rural dimension but also between regions. This condition is likely to occur because of Indonesia's highly variable geographical conditions and an archipelago with more than 16 thousand islands ${ }^{16}$. Based on this background, the aim of this study is intended to analyze the disparity between regions in health center utilization in rural areas in Indonesia. 


\section{MATERIALS AND METHODS}

The data used in this research analysis comes from the 2013 Indonesian Basic Health Survey (Riskesdas). Riskesdas was a national scale survey conducted in a cross-sectional by the Ministry of Health. Riskesdas sample was carried out by multistage cluster random sampling.

The sample framework used consists of two types, namely the sample frame for sampling the first stage and the sample frame for sampling the second stage. The first selection sample frame was the primary sampling unit (PSU) list in the sample master. The number of PSUs in the master sample was 30,000 which were selected by probability proportional to size (PPS) with the number of households resulting from the 2010 Population Census (PC2010). The PSU was a combination of several census blocks (CB) which were working areas of the PC2010 enumeration team. The PSU also features information on the number and list of names of household heads, address, level of education of the head of the household based on urban/rural area classification. The second selection sample frame was all census buildings in which there are ordinary households not including institutional household (orphanage, police/military barracks, etc.) resulting from the complete enumeration of PC2010 (PC2010-C1). Selected census buildings and households within the selected census building were updated. The update was carried out by the 2013 Riskesdas enumerator before starting to conduct interviews.

The sampling method used was a three-stage stratified sampling. The stages of this method were described as follows: The first step was to select the primary sampling unit (PSU) from the systematically selected PSU for each district/city according to the domain allocation. The second stage, from the selected PSU, 2 CB was selected by PPS with the number of households in the 2010 Population Census - Recapitulation of the number of households resulting from listing (PC2010-RBL1) in each district/city according to the domain allocation. Then one block randomly selected for Riskesdas and one census block for Susenas. The third stage, from each CB of Riskesdas, a number of census buildings $(m=25)$ were selected systematically based on the PC2010-C1 census building data.

The data was taken using a structured questionnaire ${ }^{17}$. The contents of the questionnaire consisted of information on individual characteristics (age, gender, marital status, education level, employment status, socioeconomic status, insurance, time travel, and transportation cost to health center) and health center utilization (outpatient and inpatient).

The population in this study were all adults in rural areas in Indonesia. The criteria of the respondents were residents aged 15 years and above. Respondents were considered adults at that age. The 2013 Riskesdas has been conducted with a sample of $1,027,763$ individuals. The samples analyzed in this paper were based on a unit of analysis of Indonesian adults in rural areas with 388,598 respondents. Samples were selected with inclusion criteria $\geq 15$ years old and willing to be interviewed.

The health center utilization was the use of outpatient or inpatient care to the Puskesmas. The criteria for outpatient were the utilization of the last month. While the criteria for inpatient were the utilization of the past year. This criterion was carried out assuming the respondent can still remember the occurrence of the utilization. The division of regions was grouped by the largest island. Divided into 7 regions, namely Sumatra, Kalimantan, Sulawesi, Java-Bali, Maluku Islands, Nusa Tenggara, and Papua ${ }^{18}$.

T-tests were used for age variables which were categorized as continuous variables. Chi-Square is used to test dichotomous variables. There are 8 dichotomous variables tested, namely age, sex, marital status, education level, employment status, insurance ownership, travel time, and the transportation cost to the Puskesmas. These statistical tests were to assess whether there was a statistically significant relationship between the independent variables and the Puskesmas utilization as the dependent variable. Processing data using the help of SPSS v.21 software.

The 2013 Riskesdas has an ethical permit approved by the national ethical committee (ethic number: 01.1206.207). During data collection, informed consent was used. This is by considering the aspects of procedures for data collection, voluntary, and confidentiality.

\section{RESULT}

Table 1 explains descriptively the participants in this study. It appears that participants start from the age of 15 to 128 years. The mean age of participants is 40.18 years, with Standard Deviation 16.334 . 
Table 1 Descriptive Table of The Participants $(n=388,598)$

\begin{tabular}{|c|c|c|}
\hline Variables & $\mathbf{N}$ & Percentage \\
\hline Age & 388,598 & $100 \%$ \\
\hline \multicolumn{3}{|l|}{ Gender } \\
\hline - Male $($ code $=1)$ & 188,596 & $48.5 \%$ \\
\hline - Female $($ code $=2)$ & 200,002 & $51.5 \%$ \\
\hline \multicolumn{3}{|l|}{ Marital status } \\
\hline - Single $($ code $=1)$ & 82,276 & $21.2 \%$ \\
\hline - $\quad$ Married $(\operatorname{code}=2)$ & 277,720 & $71.5 \%$ \\
\hline - $\quad$ Divorced $(\operatorname{code}=3)$ & 28,602 & $7.4 \%$ \\
\hline \multicolumn{3}{|l|}{ Education level } \\
\hline - Primary school \& under $(\operatorname{code}=1)$ & 232,779 & $59.9 \%$ \\
\hline - Junior high school (code $=2)$ & 77,177 & $19.9 \%$ \\
\hline - Senior high school ( $\operatorname{code}=3)$ & 64,488 & $16.6 \%$ \\
\hline - College $(\operatorname{code}=4)$ & 14,154 & $3.6 \%$ \\
\hline \multicolumn{3}{|l|}{ Employment status } \\
\hline - Employed $($ code $=1)$ & 243,085 & $62.6 \%$ \\
\hline - Unemployed $($ code $=2)$ & 145,513 & $37.4 \%$ \\
\hline \multicolumn{3}{|l|}{ Socioeconomic status } \\
\hline - Quintile 1 (code=1) & 116,155 & $29.9 \%$ \\
\hline - $\quad$ Quintile 2 (code $=2)$ & 98,949 & $25.5 \%$ \\
\hline - Quintile $3(\operatorname{code}=3)$ & 76,532 & $19.7 \%$ \\
\hline - Quintile $4(\operatorname{code}=4)$ & 54,969 & $14.1 \%$ \\
\hline - Quintile $5(\operatorname{code}=5)$ & 41,993 & $10.8 \%$ \\
\hline \multicolumn{3}{|l|}{ Insurance } \\
\hline - No insurance $($ code $=1)$ & 166,386 & $42.8 \%$ \\
\hline - Managed by Gov. $(\operatorname{code}=2)$ & 218,063 & $56.1 \%$ \\
\hline - Others $($ code $=3)$ & 4,149 & $1.1 \%$ \\
\hline \multicolumn{3}{|l|}{ Time travel } \\
\hline - $\leq 10$ minutes $(\operatorname{code}=1)$ & 146,412 & $37.7 \%$ \\
\hline - $\quad>10$ minutes $($ code $=2)$ & 242,186 & $62.3 \%$ \\
\hline \multicolumn{3}{|l|}{ Transportation cost } \\
\hline - $\quad \leq$ IDR $10,000($ code $=1)$ & 295,090 & $75.9 \%$ \\
\hline - $\quad>$ IDR $10,000($ code $=2)$ & 93,508 & $24.1 \%$ \\
\hline
\end{tabular}

\section{Descriptive Result}

Figure 1 explains that the main health center users are poor people. Those in the quintile 1 and 2 groups at the socioeconomic level are more likely to use health centers than other groups. This condition applies to all regions. This picture is more evident in regions in Eastern Indonesia, namely in Papua, Maluku Islands and Nusa Tenggara.

Based on Table 1, it can be seen that there is a significant difference between the region variables and all variables tested. Table 1 also explains that based on the proportion of people who use health centers, they are mostly in the Nusa Tenggara region $(8.0 \%)$. Medium age variables have the youngest average in the Papua region (36.48) and the oldest in the Java-Bali region (43.28).

Based on gender, Table 1 shows that in all regions female dominate, except for the Papua region which is dominated by male $(51.8 \%)$. Based on marital status, Table 1 shows all regions dominated by marital status of married. While based on the level of education, table 1 shows that in all regions it is dominated by society with a level of education of primary school and under. 


\section{Health Center Utilized}

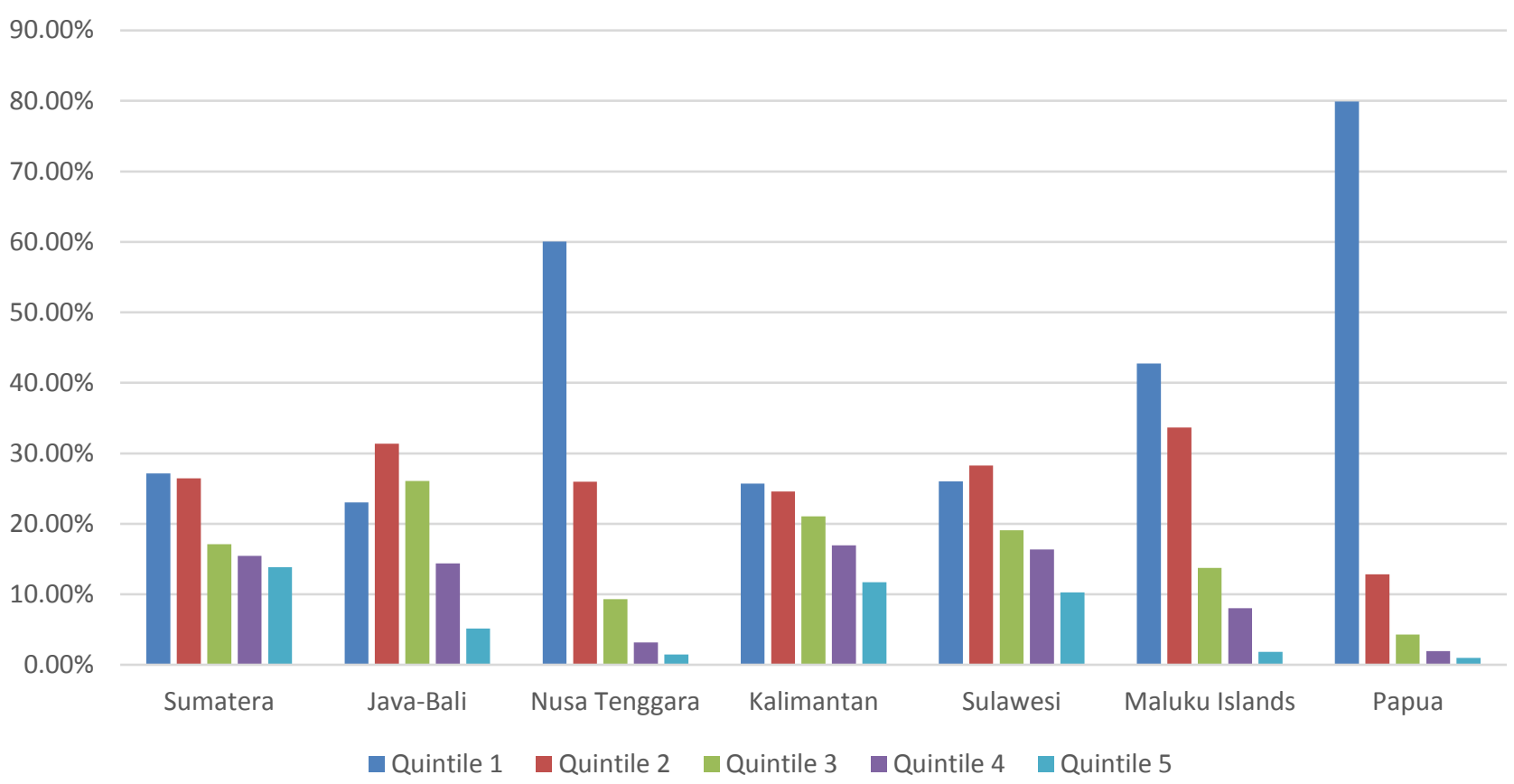

Figure 1 Distribution of health center utilized in rural Indonesia based on regions and socioeconomic status

Table 2a Descriptive Statistic of Health Center Utilization among Regions in Rural Indonesia $(n=388,598)$

\begin{tabular}{|c|c|c|c|c|c|c|c|c|c|}
\hline \multirow[b]{2}{*}{ Characteristic } & \multicolumn{7}{|c|}{ Region } & \multirow[b]{2}{*}{ All } & \multirow{2}{*}{$\begin{array}{c}\mathrm{P} \text { - } \\
\text { value }\end{array}$} \\
\hline & $\begin{array}{l}\text { Suma- } \\
\text { tera }\end{array}$ & $\begin{array}{l}\text { Java- } \\
\text { Bali }\end{array}$ & $\begin{array}{c}\text { Nusa } \\
\text { Tenggara }\end{array}$ & $\begin{array}{l}\text { Kaliman- } \\
\tan \end{array}$ & $\begin{array}{c}\text { Sulawe- } \\
\text { si }\end{array}$ & $\begin{array}{c}\text { Malu- } \\
\text { ku }\end{array}$ & Papua & & \\
\hline $\begin{array}{l}\text { Health Center } \\
\text { Utilization }\end{array}$ & & & & & & & & & $<0.001^{*}$ \\
\hline - Utilized & $\begin{array}{c}3,730 \\
3.0 \%\end{array}$ & $\begin{array}{c}4,412 \\
4.4 \%\end{array}$ & $\begin{array}{c}2,231 \\
8.0 \%\end{array}$ & $\begin{array}{c}1,512 \\
3.9 \%\end{array}$ & $\begin{array}{c}3,442 \\
5.9 \%\end{array}$ & $\begin{array}{c}770 \\
5.6 \%\end{array}$ & $\begin{array}{l}2,805 \\
12.1 \%\end{array}$ & $\begin{array}{c}18,902 \\
4.9 \%\end{array}$ & \\
\hline - Not utilized & $\begin{array}{c}122,167 \\
97.0 \%\end{array}$ & $\begin{array}{l}95,860 \\
95.6 \%\end{array}$ & $\begin{array}{c}25,654 \\
92.0 \%\end{array}$ & $\begin{array}{c}37,483 \\
96.1 \%\end{array}$ & $\begin{array}{c}55,350 \\
94.1 \%\end{array}$ & $\begin{array}{c}12,878 \\
94.4 \%\end{array}$ & $\begin{array}{c}20,304 \\
87.9 \%\end{array}$ & $\begin{array}{c}369,696 \\
95.1 \%\end{array}$ & \\
\hline Age (mean) & $\begin{array}{r}125,897 \\
(38.58)\end{array}$ & $\begin{array}{c}100,272 \\
(43.28)\end{array}$ & $\begin{array}{l}27,885 \\
(40.53)\end{array}$ & $\begin{array}{l}38,995 \\
(38.86)\end{array}$ & $\begin{array}{l}58,792 \\
(40.65)\end{array}$ & $\begin{array}{l}13,648 \\
(39.29)\end{array}$ & $\begin{array}{l}23,109 \\
(36.48)\end{array}$ & $\begin{array}{c}388,598 \\
(40.18)\end{array}$ & $<0.001^{*}$ \\
\hline Gender & & & & & & & & & $<0.001^{*}$ \\
\hline - Male & $\begin{array}{l}62,187 \\
49.4 \%\end{array}$ & $\begin{array}{l}47,534 \\
47.4 \%\end{array}$ & $\begin{array}{l}13,216 \\
47.4 \%\end{array}$ & $\begin{array}{c}19,282 \\
49.4 \%\end{array}$ & $\begin{array}{c}27,930 \\
47.5 \%\end{array}$ & $\begin{array}{l}6,473 \\
47.4 \%\end{array}$ & $\begin{array}{c}11,974 \\
51.8 \%\end{array}$ & $\begin{array}{c}188,596 \\
48.5 \%\end{array}$ & \\
\hline - Female (Ref.) & $\begin{array}{l}63,710 \\
50.6 \%\end{array}$ & $\begin{array}{l}52,738 \\
52.6 \%\end{array}$ & $\begin{array}{l}14,669 \\
52.6 \%\end{array}$ & $\begin{array}{c}19,713 \\
50.6 \%\end{array}$ & $\begin{array}{c}30,862 \\
52.5 \%\end{array}$ & $\begin{array}{l}7,175 \\
52.6 \%\end{array}$ & $\begin{array}{c}11,135 \\
48.2 \%\end{array}$ & $\begin{array}{c}200,002 \\
51.5 \%\end{array}$ & \\
\hline Marital status & & & & & & & & & $<0.001^{*}$ \\
\hline - Single & $\begin{array}{l}31,921 \\
25.4 \%\end{array}$ & $\begin{array}{l}16,641 \\
16.6 \%\end{array}$ & $\begin{array}{l}6,531 \\
23.4 \%\end{array}$ & $\begin{array}{l}7,840 \\
20.1 \%\end{array}$ & $\begin{array}{l}12,520 \\
21.3 \%\end{array}$ & $\begin{array}{l}2,893 \\
21.2 \%\end{array}$ & $\begin{array}{l}3,930 \\
17.0 \%\end{array}$ & $\begin{array}{c}82,276 \\
21.2 \%\end{array}$ & \\
\hline - Married & $\begin{array}{l}85,728 \\
68.1 \%\end{array}$ & $\begin{array}{l}73,938 \\
73.7 \%\end{array}$ & $\begin{array}{l}19,780 \\
70.9 \%\end{array}$ & $\begin{array}{c}28,492 \\
73.1 \%\end{array}$ & $\begin{array}{c}41,625 \\
70.8 \%\end{array}$ & $\begin{array}{l}10,037 \\
73.5 \%\end{array}$ & $\begin{array}{l}18,120 \\
78.4 \%\end{array}$ & $\begin{array}{c}277,720 \\
71.5 \%\end{array}$ & \\
\hline - Divorce (Ref.) & $\begin{array}{c}8,248 \\
6.6 \%\end{array}$ & $\begin{array}{l}9,693 \\
9.7 \%\end{array}$ & $\begin{array}{c}1,574 \\
5.6 \%\end{array}$ & $\begin{array}{c}2,663 \\
6.8 \%\end{array}$ & $\begin{array}{c}4,647 \\
7.9 \%\end{array}$ & $\begin{array}{c}718 \\
5.3 \%\end{array}$ & $\begin{array}{c}1,059 \\
4.6 \%\end{array}$ & $\begin{array}{c}28,602 \\
7.4 \%\end{array}$ & \\
\hline
\end{tabular}


Table 2b Descriptive Statistic of Health Center Utilization among Regions in Rural Indonesia $(n=388,598)$

\begin{tabular}{|c|c|c|c|c|c|c|c|c|c|}
\hline \multirow[b]{2}{*}{ Characteristic } & \multicolumn{7}{|c|}{ Region } & \multirow[b]{2}{*}{ All } & \multirow[b]{2}{*}{$\begin{array}{c}\text { P- } \\
\text { value }\end{array}$} \\
\hline & $\begin{array}{l}\text { Suma- } \\
\text { tera }\end{array}$ & $\begin{array}{l}\text { Java- } \\
\text { Bali }\end{array}$ & $\begin{array}{c}\text { Nusa } \\
\text { Tenggara }\end{array}$ & $\begin{array}{l}\text { Kaliman- } \\
\text { tan }\end{array}$ & $\begin{array}{c}\text { Sulawe- } \\
\text { si }\end{array}$ & $\begin{array}{c}\text { Malu- } \\
\text { ku }\end{array}$ & Papua & & \\
\hline Education level & & & & & & & & & $<0.001^{*}$ \\
\hline $\begin{array}{l}\text { - Primary sch. \& } \\
\text { under }\end{array}$ & 64,969 & 68,676 & 18,840 & 24,042 & 34,137 & 7,294 & 14,821 & 232,779 & \\
\hline - Junior high sch. & $\begin{array}{c}51.6 \% \\
29,192 \\
23.2 \%\end{array}$ & $\begin{array}{c}68.5 \% \\
17,433 \\
17.4 \%\end{array}$ & $\begin{array}{l}67.6 \% \\
4,305 \\
15.4 \%\end{array}$ & $\begin{array}{l}61.7 \% \\
7,689 \\
19.7 \%\end{array}$ & $\begin{array}{c}58.1 \% \\
11,696 \\
19.9 \%\end{array}$ & $\begin{array}{l}53.4 \% \\
2,991 \\
21.9 \%\end{array}$ & $\begin{array}{l}64.1 \% \\
3,871 \\
16.8 \%\end{array}$ & $\begin{array}{c}59.9 \% \\
77,177 \\
19.9 \%\end{array}$ & \\
\hline - Senior high sch. & $\begin{array}{c}26,779 \\
21.3 \%\end{array}$ & $\begin{array}{c}11,678 \\
11.6 \%\end{array}$ & $\begin{array}{l}3,774 \\
13.5 \%\end{array}$ & $\begin{array}{l}5,855 \\
15.0 \%\end{array}$ & $\begin{array}{l}10,244 \\
17.4 \%\end{array}$ & $\begin{array}{l}2,728 \\
20.0 \%\end{array}$ & $\begin{array}{l}3,430 \\
14.8 \%\end{array}$ & $\begin{array}{c}64,488 \\
16.6 \%\end{array}$ & \\
\hline - College (Ref.) & $\begin{array}{c}4,957 \\
3.9 \%\end{array}$ & $\begin{array}{c}2,485 \\
2.5 \%\end{array}$ & $\begin{array}{c}966 \\
3.5 \%\end{array}$ & $\begin{array}{c}1,409 \\
3.6 \%\end{array}$ & $\begin{array}{c}2,715 \\
4.6 \%\end{array}$ & $\begin{array}{l}635 \\
4.7 \%\end{array}$ & $\begin{array}{c}987 \\
4.3 \%\end{array}$ & $\begin{array}{c}14,154 \\
3.6 \%\end{array}$ & \\
\hline $\begin{array}{l}\text { Employment } \\
\text { status }\end{array}$ & & & & & & & & & $<0.001^{*}$ \\
\hline - Employed & $\begin{array}{c}79,050 \\
62.8 \%\end{array}$ & $\begin{array}{c}64,697 \\
64.5 \%\end{array}$ & $\begin{array}{l}19,057 \\
68.3 \%\end{array}$ & $\begin{array}{l}25,357 \\
65.0 \%\end{array}$ & $\begin{array}{c}31,637 \\
53.8 \%\end{array}$ & $\begin{array}{l}7,866 \\
57.6 \%\end{array}$ & $\begin{array}{c}15,421 \\
66.7 \%\end{array}$ & $\begin{array}{c}243,085 \\
62.6 \%\end{array}$ & \\
\hline - Unemployed & $\begin{array}{c}46,847 \\
37.2 \%\end{array}$ & $\begin{array}{c}35,575 \\
35.5 \%\end{array}$ & $\begin{array}{l}8,828 \\
31.7 \%\end{array}$ & $\begin{array}{l}13,638 \\
35.0 \%\end{array}$ & $\begin{array}{c}27,155 \\
46.2 \%\end{array}$ & $\begin{array}{l}5,782 \\
42.4 \%\end{array}$ & $\begin{array}{l}7,688 \\
33.3 \%\end{array}$ & $\begin{array}{c}145,513 \\
37.4 \%\end{array}$ & \\
\hline $\begin{array}{l}\text { Socioeconomic } \\
\text { status }\end{array}$ & & & & & & & & & $<0.001^{*}$ \\
\hline - Quintile 1 & $\begin{array}{c}29,269 \\
23.2 \%\end{array}$ & $\begin{array}{c}20,609 \\
20.6 \%\end{array}$ & $\begin{array}{l}15,760 \\
56.5 \%\end{array}$ & $\begin{array}{l}10,847 \\
27.8 \%\end{array}$ & $\begin{array}{l}17,185 \\
29.2 \%\end{array}$ & $\begin{array}{l}6,118 \\
44.8 \%\end{array}$ & $\begin{array}{l}16,367 \\
70.8 \%\end{array}$ & $\begin{array}{c}116,155 \\
29.9 \%\end{array}$ & \\
\hline - Quintile 2 & $\begin{array}{c}30,017 \\
23.8 \%\end{array}$ & $\begin{array}{c}28,907 \\
28.8 \%\end{array}$ & $\begin{array}{l}7,119 \\
25.5 \%\end{array}$ & $\begin{array}{l}9,773 \\
25.1 \%\end{array}$ & $\begin{array}{l}15,265 \\
26.0 \%\end{array}$ & $\begin{array}{l}4,106 \\
30.1 \%\end{array}$ & $\begin{array}{l}3,762 \\
16.3 \%\end{array}$ & $\begin{array}{c}98,949 \\
25.5 \%\end{array}$ & \\
\hline - Quintile 3 & $\begin{array}{c}24,978 \\
19.8 \%\end{array}$ & $\begin{array}{c}25,751 \\
25.7 \%\end{array}$ & $\begin{array}{l}2,935 \\
10.5 \%\end{array}$ & $\begin{array}{l}7,878 \\
20.2 \%\end{array}$ & $\begin{array}{c}11,326 \\
19.3 \%\end{array}$ & $\begin{array}{l}2,126 \\
15.6 \%\end{array}$ & $\begin{array}{c}1,538 \\
6.7 \%\end{array}$ & $\begin{array}{c}76,532 \\
19.7 \%\end{array}$ & \\
\hline - Quintile 4 & $\begin{array}{c}20,339 \\
16.2 \%\end{array}$ & $\begin{array}{c}16,717 \\
16.7 \%\end{array}$ & $\begin{array}{c}1,448 \\
5.2 \%\end{array}$ & $\begin{array}{l}5,759 \\
14.8 \%\end{array}$ & $\begin{array}{l}8,686 \\
14.8 \%\end{array}$ & $\begin{array}{l}1,016 \\
7.4 \%\end{array}$ & $\begin{array}{c}1,004 \\
4.3 \%\end{array}$ & $\begin{array}{c}54,969 \\
14.1 \%\end{array}$ & \\
\hline - Quintile 5 (Ref.) & $\begin{array}{c}21,294 \\
16.9 \%\end{array}$ & $\begin{array}{l}8,288 \\
8.3 \%\end{array}$ & $\begin{array}{c}623 \\
2.2 \%\end{array}$ & $\begin{array}{l}4,738 \\
12.2 \%\end{array}$ & $\begin{array}{l}6,330 \\
10.8 \%\end{array}$ & $\begin{array}{c}282 \\
2.1 \%\end{array}$ & $\begin{array}{l}438 \\
1.9 \%\end{array}$ & $\begin{array}{c}41,993 \\
10.8 \%\end{array}$ & \\
\hline $\begin{array}{l}\text { Insurance } \\
\text { ownership }\end{array}$ & & & & & & & & & $<0.001^{*}$ \\
\hline - No insurance & $\begin{array}{c}56,741 \\
45.1 \%\end{array}$ & $\begin{array}{c}51,068 \\
50.9 \%\end{array}$ & $\begin{array}{l}9,191 \\
33.0 \%\end{array}$ & $\begin{array}{l}19,296 \\
49.5 \%\end{array}$ & $\begin{array}{c}18,029 \\
30.7 \%\end{array}$ & $\begin{array}{l}5,637 \\
41.3 \%\end{array}$ & $\begin{array}{l}6,424 \\
27.8 \%\end{array}$ & $\begin{array}{c}166,386 \\
42.8 \%\end{array}$ & \\
\hline - Managed by Gov. & $\begin{array}{c}67,294 \\
53.5 \%\end{array}$ & $\begin{array}{c}48,640 \\
48.5 \%\end{array}$ & $\begin{array}{c}18,605 \\
66.7 \%\end{array}$ & $\begin{array}{l}18,801 \\
48.2 \%\end{array}$ & $\begin{array}{c}40,544 \\
69.0 \%\end{array}$ & $\begin{array}{l}7,965 \\
58.4 \%\end{array}$ & $\begin{array}{l}16,214 \\
70.2 \%\end{array}$ & $\begin{array}{c}218,063 \\
56.1 \%\end{array}$ & \\
\hline - Others (Ref.) & $\begin{array}{c}1,862 \\
1.5 \%\end{array}$ & $\begin{array}{c}564 \\
0.6 \%\end{array}$ & $\begin{array}{c}89 \\
0.3 \%\end{array}$ & $\begin{array}{l}898 \\
2.3 \%\end{array}$ & $\begin{array}{c}219 \\
0.4 \%\end{array}$ & $\begin{array}{c}46 \\
0.3 \%\end{array}$ & $\begin{array}{c}471 \\
2.0 \%\end{array}$ & $\begin{array}{c}4,149 \\
1.1 \%\end{array}$ & \\
\hline $\begin{array}{l}\text { Time travel } \\
\bullet \leq 10 \text { minute }\end{array}$ & $\begin{array}{c}45,372 \\
36.0 \%\end{array}$ & $\begin{array}{c}33,028 \\
32.9 \%\end{array}$ & $\begin{array}{l}9,805 \\
35.2 \%\end{array}$ & $\begin{array}{c}17,144 \\
44.0 \%\end{array}$ & $\begin{array}{c}23,847 \\
40.6 \%\end{array}$ & $\begin{array}{l}7,285 \\
53.4 \%\end{array}$ & $\begin{array}{l}9,931 \\
43.0 \%\end{array}$ & $\begin{array}{c}146,412 \\
37.7 \%\end{array}$ & $<0.001^{*}$ \\
\hline - $>10$ minutes & $\begin{array}{c}80,525 \\
64.0 \% \\
\end{array}$ & $\begin{array}{c}67,244 \\
67.1 \% \\
\end{array}$ & $\begin{array}{l}18,080 \\
64.8 \% \\
\end{array}$ & $\begin{array}{r}21,851 \\
56.0 \% \\
\end{array}$ & $\begin{array}{c}34,945 \\
59.4 \% \\
\end{array}$ & $\begin{array}{l}6,363 \\
46.6 \% \\
\end{array}$ & $\begin{array}{c}13,178 \\
57.0 \% \\
\end{array}$ & $\begin{array}{c}242,186 \\
62.3 \% \\
\end{array}$ & \\
\hline $\begin{array}{l}\text { Transportation } \\
\text { cost }\end{array}$ & & & & & & & & & $<0.001^{*}$ \\
\hline$\bullet \leq \operatorname{IDR} 10,000$ & $\begin{array}{l}91,231 \\
72.5 \%\end{array}$ & $\begin{array}{c}83,146 \\
82.9 \%\end{array}$ & $\begin{array}{c}20,716 \\
74.3 \%\end{array}$ & $\begin{array}{l}27,321 \\
70.1 \%\end{array}$ & $\begin{array}{l}46,638 \\
79.3 \%\end{array}$ & $\begin{array}{l}9,458 \\
69.3 \%\end{array}$ & $\begin{array}{l}16,580 \\
71.7 \%\end{array}$ & $\begin{array}{c}295,090 \\
75.9 \%\end{array}$ & \\
\hline - > IDR 10,000 & $\begin{array}{l}34,666 \\
27.5 \%\end{array}$ & $\begin{array}{c}17,126 \\
17.1 \%\end{array}$ & $\begin{array}{l}7,169 \\
25.7 \%\end{array}$ & $\begin{array}{l}11,674 \\
29.9 \%\end{array}$ & $\begin{array}{l}12,154 \\
20.7 \%\end{array}$ & $\begin{array}{l}4,190 \\
30.7 \%\end{array}$ & $\begin{array}{l}6,529 \\
28.3 \%\end{array}$ & $\begin{array}{l}93,508 \\
24.1 \%\end{array}$ & \\
\hline
\end{tabular}

Note: Chi-Square test was used for dichotomous variables, and T-test for continuous variables; *Significant at level $95 \%$.

Table $2 a$ shows that based on working status is dominated by those who have jobs, with the largest proportion in the Nusa Tenggara region (68.3\%). Based on socioeconomic conditions, those who live in the East are more dominated by the poor (quintile
1 and 2), especially in the Papua region, Maluku and Nusa Tenggara. Table $2 \mathrm{~b}$ shows that based on insurance ownership is dominated by those who have insurance managed by the government (Askes, Jamkesmas, Jamkesda, Jamsostek), except Java- 
Bali and Kalimantan regions which are dominated by those who do not have insurance.

Table $2 \mathrm{~b}$ shows based on the time needed to reach the health center dominated by the category "> 10 minutes". However, based on the transportation costs needed to reach the health center, it was dominated by the "cost of IDR 10,000" transportation cost category. The biggest proportion is in the Java-Bali region (82.9\%).

Multivariate Regression Analyses

Table 3 Binary Logistic Regression of Health Center Utilization among Regions in Rural Indonesia $(n=388,598)$

\begin{tabular}{|c|c|c|c|c|}
\hline \multirow{2}{*}{ Predictor } & \multicolumn{4}{|c|}{ Health Center Utilization } \\
\hline & Sig. & OR & Lower Bound & Upper Bound \\
\hline Region: Sumatera & $<0.001^{*}$ & 3.781 & 3.580 & 3.993 \\
\hline Region: Java-Bali & $<0.001^{*}$ & 2.773 & 2.627 & 2.927 \\
\hline Region: Nusa Tenggara & $<0.001^{*}$ & 1.582 & 1.490 & 1.679 \\
\hline Region: Kalimantan & $<0.001^{*}$ & 2.832 & 2.648 & 3.030 \\
\hline Region: Sulawesi & $<0.001^{*}$ & 2.254 & 2.133 & 2.382 \\
\hline Region: Maluku & $<0.001^{*}$ & 2.175 & 1.999 & 2.366 \\
\hline Age & $<0.001^{*}$ & 0.994 & 0.992 & 0.995 \\
\hline Gender: Male & $<0.001^{*}$ & 1.341 & 1.297 & 1.387 \\
\hline Marital Status: single & $<0.001^{*}$ & 1.737 & 1.603 & 1.882 \\
\hline Marital Status: married & 0.083 & 1.050 & 0.994 & 1.109 \\
\hline Education: under primary school & 0.262 & 0.952 & 0.873 & 1.038 \\
\hline Education: junior high school & 0.286 & 0.952 & 0.869 & 1.042 \\
\hline Education: senior high school & 0.558 & 0.973 & 0.888 & 1.066 \\
\hline Employment status: Employed & $<0.001^{*}$ & 1.091 & 1.054 & 1.129 \\
\hline Socioeconomic: quintile 1 & $<0.001^{*}$ & 0.698 & 0.654 & 0.745 \\
\hline Socioeconomic: quintile 2 & $<0.001^{*}$ & 0.743 & 0.696 & 0.793 \\
\hline Socioeconomic: quintile 3 & $<0.001^{*}$ & 0.808 & 0.756 & 0.864 \\
\hline Socioeconomic: quintile 4 & $<0.001^{*}$ & 0.820 & 0.764 & 0.879 \\
\hline Insurance ownership: No insurance & 0.966 & 0.996 & 0.812 & 1.221 \\
\hline Insurance: Managed by Gov. & $<0.001^{*}$ & 0.482 & 0.393 & 0.590 \\
\hline Travel time: $\leq 10$ minutes & $<0.001^{*}$ & 0.917 & 0.889 & 0.945 \\
\hline Transportation cost: $\leq$ IDR 10,000 & $<0.001^{*}$ & 0.551 & 0.528 & 0.574 \\
\hline
\end{tabular}

Note: The reference category is "Not Utilized"; 95\% Confidence Interval for OR; *Significant at level 95\%.

Table 3 represents the results of a binary logistic test. The results express that there are statistically significant disparities between regions. All regions show better utilization than the Papua region as a reference. The best utilization is in the Sumatra region, which is 3.781 times more utilizing health centers than the Papua region $(\mathrm{OR}=3.781 ; 95 \% \mathrm{Cl}=$ 3.580-3.993). The utilization of health center which was slightly different from the Papua region was the Nusa Tenggara region $(\mathrm{OR}=1.582 ; 95 \% \mathrm{Cl}=1.490$ $1.679)$ and the Maluku region $(\mathrm{OR}=2.175 ; 95 \%$ 1.999-2.366). All three regions are all in the Eastern part of Indonesia.

Table 3 indicates that male had 1.341 times better utilization than female $(\mathrm{OR}=1.341 ; 95 \% \mathrm{Cl}=1.297$ 1.387). Those who have the marital status of singles have health center utilization 1.737 times better than those divorced. While based on the level of education, no significant differences were found between levels of education in communities in rural Indonesia.

Table 3 shows that those who were employed 1.091 times were more likely to use health centers than those who were unemployed. Based on the socioeconomic level, no group has better health center utilization than the richest (quintile 5) group in rural Indonesia. Those in the poorest group (quintile 1 ) used the health center 0.698 times the richest group $(\mathrm{OR}=0.698 ; 95 \% \mathrm{Cl}=0.654-0.745)$.

\section{DISCUSSION}

The results of the study show that there are gaps between regions in the utilization of Puskesmas in 
rural Indonesia. The geographical conditions of Indonesia and the disparity in urban-rural development are indeed very possible for disparities in the use of health centers. Geographical conditions in the form of islands make some small and remote islands very difficult to reach. This is also influenced by the availability of regular transportation to these remote islands ${ }^{10,19}$. Several other studies on spatial health service disparities in several countries were also found to have the same

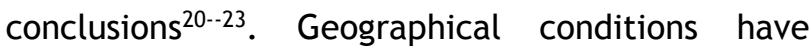
proven to contribute significantly to the disparity between regions.

The results showed that the utilization of health centers in the West tends to be better than in the East. This condition is directly proportional to economic development in Indonesia, which indeed shows inequality between the West and East. Development in the East region tends to lag behind other regions (24)(25)(1), including health development ${ }^{4,26}$.

As a single variable, low socioeconomic status (quintile 1 and 2) has the dominant proportion of health center utilization (see Figure 1), while in a multivariate manner, a group with high socioeconomic status (quintile 5 ) actually has better health center utilization. This shows that high socioeconomic groups are more aware of utilizing their health rights because they have relatively better knowledge ${ }^{4}$. Rich people who are knowledgeable are smarter in taking advantage of opportunities. The results of this study are in line with several studies related to socioeconomic in developing countries ${ }^{27}$, and also other countries, namely USA ${ }^{28}$, Bangladesh ${ }^{29}$, Lao People's Democratic Republic ${ }^{30}$, and in several European countries ${ }^{31}$.

Those who need more time to the health center (> 10 minutes) and more expensive transportation costs (> IDR 10,000; around \$1) have better health center utilization. This result is the impact of the low service tariff policy at the Puskesmas. Even in some regions, the local government actually frees the community to utilize the Puskesmas as a basic service $^{2,32,33}$.

Limitations in this study can only detect disparities that occur between regions only superficially. Further studies are needed that can detect how these disparities can occur.

\section{CONCLUSIONS}

Based on the research results and discussion it can be concluded that there is a proven disparity in health center utilization between regions in rural Indonesia. Regions in the western part of Indonesia tend to have better health center utilization in rural areas. The disparity in health center utilization is also found in other categories, namely gender, marital status, employment status, socioeconomic level, insurance ownership, travel time and transportation costs to the health center. Structured policies are needed to reach rural communities. The results of this study can be used as a reference for making policies that focus on equality of services to reduce existing disparities.

\section{ACKNOWLEDGMENTS}

The author would like to thank the National Institute of Health Research and Development, Ministry of Health, who has agreed to allow the 2013 Riskesdas data to be analyzed in this article.

\section{DECLARATION OF CONFLICTING INTERESTS}

The authors declared no potential conflicts of interest with respect to the research, authorship, and/or publication of this article.

\section{FUNDING}

No funding sources.

\section{REFERENCES}

1. Mubasyiroh R, Nurhotimah E, Laksono AD. Indeks Aksesibilitas Pelayanan Kesehatan di Indonesia. In: Supriyanto S, Chalidyanto D, Wulandari RD, editors. Aksesibilitas Pelayanan Kesehatan di Indonesia. Jogjakarta: PT Kanisius; 2016. p. 21-58.

2. Megatsari H, Laksono AD, Ridlo IA, Yoto $M$, Azizah AN. Community Perspective about Health Services Access. Bul Penelit Sist Kesehat. 2018;21:247-253.

3. Laksono AD, Nantabah ZK, Wulandari RD. Access Barriers to Health Center for Elderly in Indonesia. Bul Penelit Sist Kesehat. 2018;21(4):228-35.

4. Suparmi, Kusumawardani N, Nambiar D, Trihono, Hosseinpoor AR. Subnational regional inequality in the public health development index in Indonesia. Glob Health Action. 2018;11(1).

5. Park YJ, Martin EG. Geographic Disparities in Access to Nursing Home Services: Assessing Fiscal Stress and Quality of Care. Health Serv Res. 2018;53:2932-51.

6. Li J, Shi L, Liang H, Ding G, Xu L. Urban-rural disparities in health care utilization among 
Chinese adults from 1993 to 2011. BMC Health Serv Res. 2018;18(102):1-9.

7. Ault-Brutus A, Alegria M. Racial/ethnic differences in perceived need for mental health care and disparities in use of care among those with perceived need in 19901992 and 2001-2003. Ethn Heal. 2018;23(2):142-57.

8. Johar M, Soewondo P, Pujisubekti R, Satrio $\mathrm{HK}$, Adji A. Inequality in access to health care, health insurance and the role of supply factors. Soc Sci Med. 2018;213:134-45.

9. Dewi A, Mukti AG. The strategy to achieve universal health coverage membership in Indonesia. Res J Pharm Technol. 2018;11(5):1774-7.

10. Laksono $A D$, Wulandari RD, Soedirham $O$. Urban and Rural Disparities in Hospital Utilization among Indonesian Adults. Iran J Public Health [Internet]. 2019;48(2):247-55. Available from: http://ijph.tums.ac.ir/index.php/ijph/artic le/view/16143

11. Wang S, Kou C, Liu Y, Li B, Tao Y, D’Arcy C, et al. Rural-Urban Differences in the Prevalence of Chronic Disease in Northeast China. Asia-Pacific J Public Heal. 2014;1-13.

12. Cheng $L$, Tan L, Zhang L, Wei S, Liu L, Long $L$, et al. Chronic disease mortality in rural and urban residents in Hubei Province, China, 2008-2010. BMC Public Health. 2013;13(1).

13. Febriawati H, Alfansi L, Hadi ED, Ab SA. The role of management function to the achievement of Puskesmas indicator as a gatekeeper of national health guarantee in Bengkulu City. Indian J Public Heal Res Dev. 2018;9(9):353-7.

14. Hermansyah A, Sainsbury E, Krass I. Investigating the impact of the universal healthcare coverage programme on community pharmacy practice. Heal Soc Care Community. 2018;26(2):e249-60.

15. Istikmal, Wibowo TA, Yovita LV. Polygon WebGIS of distric level for development and monitoring of PUSKESMAS in health care services. In: Proceeding of 2015 1st International Conference on Wireless and Telematics, ICWT 2015. Manado: Institute of Electrical and Electronics Engineers Inc.; 2016.
16. United Nations Group of Experts on Geographical Names. United Nations Conference on the Standardization of Geographical Names, 11th [Internet]. 2017 [cited 2018 Sep 1]. Available from: https://unstats.un.org/unsd/geoinfo/UNGE GN/ungegnConf11.html

17. National Institute of Health Research and Development of Ministry of Health of the Republic of Indonesia. The 2013 Indonesia Basic Health Survey (Riskesdas): National Report. Jakarta; 2013.

18. Kusumawardani N, Tarigan I, Suparmia, Schlotheuber A. Socio-economic, demographic and geographic correlates of cigarette smoking among Indonesian adolescents: results from the 2013 Indonesian Basic Health Research (RISKESDAS) survey. Glob Health Action. 2018;11.

19. Suharmiati, Laksono AD, Astuti WD. Review Kebijakan tentang Pelayanan Kesehatan Puskesmas di Daerah Terpencil Perbatasan. Bul Penelit Sist Kesehat. 2013;16(2):109116.

20. O'Donnell TFX, Powell C, Deery SE, Darling JD, Hughes K, Giles K., et al. Regional variation in racial disparities among patients with peripheral artery disease. J Vasc Surg. 2018;68(2):519-26.

21. Tyler PD, Stone DJ, Geisler BP, McLennan S, Celi LA, Rush B. Racial and Geographic Disparities in Interhospital ICU Transfers. Crit Care Med. 2018;46(1):e76-80.

22. Rostami M, Karamouzian $M$, Khosravi A, Rezaeian S. Gender and geographical inequalities in fatal drug overdose in Iran: A province-level study in 2006 and 2011. Spat Spatiotemporal Epidemiol. 2018;25:19-24.

23. Momenyan S, Kavousi A, Poorolajal J, Momenyan $\mathrm{N}$. Spatial inequalities and predictors of HIV/AIDS mortality risk in Hamadan, Iran: a retrospective cohort study. Epidemiol Health. 2018;40.

24. Yudhistira MH, Sofiyandi Y. Seaport status, port access, and regional economic development in Indonesia. Marit Econ Logist. 2018;20(4):549-68.

25. Indra I, Nazara S, Hartono D, Sumarto S. Expenditure inequality and polarization in 
Indonesia, 2002-2012. Int J Soc Econ. 2018;45(10):1469-86

26. Afifah T, Nuryetty MT, Cahyorini, Musadad DA, Schlotheuber A, Bergen $N$, et al. Subnational regional inequality in access to improved drinking water and sanitation in Indonesia: results from the 2015 Indonesian National Socioeconomic Survey (SUSENAS). Glob Health Action. 2018;11.

27. Amo-Adjei J, Aduo-Adjei K, Opoku-Nyamah C, Izugbara C. Analysis of socioeconomic differences in the quality of antenatal services in low and middle-income countries (LMICs). PLoS One. 2018;13(2).

28. Yuan Y, Louis C, Cabral H, Schneider JC, Ryan CM, Kazis LE. Socioeconomic and Geographic Disparities in Accessing Nursing Homes With High Star Ratings. J Am Med Dir Assoc. 2018;

29. Boulton ML, Carlson BF, Power LE, Wagner AL. Socioeconomic factors associated with full childhood vaccination in Bangladesh, 2014. Int J Infect Dis. 2018;69:35-40.

30. Do N, Tran HTG, Phonvisay A, Oh J. Trends of socioeconomic inequality in using maternal health care services in Lao People's Democratic Republic from year 2000 to 2012. BMC Public Health. 2018;18(1).

31. Doganis D, Panagopoulou P, Tragiannidis A, Vichos T, Moschovi M, Polychronopoulou S, et al. Survival and mortality rates of Wilms tumour in Southern and Eastern European countries: Socioeconomic differentials compared with the United States of America. Eur J Cancer. 2018;101:38-46.

32. Gumilang MF, Eng $\mathrm{KI}$, Galinium $M$. Assessment of service maturity of "Kartu Jakarta Sehat" application system. In: 2013 International Conference on Information Technology and Electrical Engineering: "Intelligent and Green Technologies for Sustainable Development", ICITEE 2013. IEEE Computer Society; 2013. p. 25-30.

33. Kurniawan MF, Harbianto D, Siswoyo BE, Susilo D, Trisnantoro L, Ernawaty, et al. Analisis Bottom-Up Pembiayaan Kesehatan di Puskesmas di Provinsi Jawa Timur dan Nusa Tenggara Timur. In: Indonesia Health Economic Association Conference. Bandung; 2014. 